\title{
Substance $P$ and Calcitonin Gene-Related Peptide in the Glands of External Auditory Canal Skin
}

\author{
Yong Joo Yoon ${ }^{1} \cdot$ Jin Yoon ${ }^{2}$ Eun Jung Lee ${ }^{1}$ Jong Seung Kim \\ 'Department of Otolaryngology, Chonbuk National University Medical School and Research Institute of Chonbuk National University Hospital, \\ Jeonju; '2Department of Otolaryngology, Wonkwang University Hospital, Iksan, Korea
}

Objectives. The earwax (cerumen) that covers external auditory canal (EAC) skin contains a mixture of ceruminous and sebaceous gland substances, such as lipids, peptides, and proteins. The components secreted from the ceruminous gland that is a modified sweat gland form cerumen and contain several antimicrobial factors. Since substance P (SP) and calcitonin gene-related peptide (CGRP), known as a secretagogue, have been found in sweat glands, our purpose was to determine the expression of SP and CGRP in the glands of EAC skin.

Methods. Sections of normal human EAC skins were immunostained for the presence of SP and CGRP using polyclonal antibodies. Immunoreactivity was detected using an avidin-biotin peroxidase complex method.

Results. SP staining was found in ceruminous gland acini and myoepithelial cells. But the SP staining was not found in the sebaceous glands and epidermal region. CGRP was strongly stained in the ceruminous gland and weakly in the sebaceous gland cells. Interestingly, most prominent staining of SP and CGRP was noted in the myoepithelial cells of the ceruminous gland.

Conclusion. The findings in this study suggest that SP and CGRP are expressed in the glands of the EAC skin and secreted in the process of ceruminous gland secretion.

Keywords. Substance P; Calcitonin Gene-Related Peptide; External Auditory Canal; Gland

\section{INTRODUCTION}

The ceruminous gland is a modified sweat gland and plays a major role in formation of cerumen (earwax) in the external auditory canal (EAC) [1]. The sweat glands of EAC skin secrete a wide variety of antimicrobial proteins and peptides, such as lysozyme, lactoferrin, and $\beta$-defensins [2-6]. Gland secretion is neurally regulated by acetylcholine, substance $\mathrm{P}$ (SP), calcitonin gene-related peptide (CGRP), vasoactive intestinal peptide, and other components [7]. Among them, SP and CGRP play a major role in the gland secretion induced by harmful stimuli [8]. In preliminary studies, we have published reports about the expres-

- Received January 31, 2017

Revised May 16, 2017

Accepted May 26, 2017

- Corresponding author: Jin Yoon

Department of Otolaryngology, Wonkwang University Hospital,

895 Muwang-ro, Iksan 54538, Korea

Tel: +82-63-859-1445, Fax: +82-63-841-6556

E-mail: sverige80@naver.com sion of antimicrobial proteins and peptides in the cerumen and the ceruminous glands. Therefore, it is conceivable that the interrelationship of antimicrobial factors and secretagogues such as SP and CGRP has clinical significance in their essential protective roles for the harmful states. However, the mechanism involved in the secretion has not been elucidated. Moreover, little is known about the expression of SP and CGRP in the EAC skin.

In the present study, we examined distribution patterns of SP and CGRP in human EAC skin, especially its relation with ceruminous glands using antibodies and the avidin-biotin peroxidase complex method.

\section{MATERIALS AND METHODS}

\section{Tissue specimens}

Skin from cartilaginous EAC was obtained from 10 adult individuals who had ear surgery (canaloplasty) and patients with canal cholesteatoma, otitis externa, previous ear surgery, or any

Copyright () 2017 by Korean Society of Otorhinolaryngology-Head and Neck Surgery.

This is an open-access article distributed under the terms of the Creative Commons Attribution Non-Commercial License (http://creativecommons.org/licenses/by-nc/4.0)

which permits unrestricted non-commercial use, distribution, and reproduction in any medium, provided the original work is properly cited. 
disease related to immunologic problems were excluded. The specimens were immediately fixed in a freshly prepared fixative containing $4 \%$ paraformaldehyde in phosphate buffer at $\mathrm{pH} 7.4$ overnight. All samples were then embedded in paraffin blocks, sectioned $4 \mu \mathrm{m}$ thickness, placed on albumin-coated glass slides, deparaffinized with xylene, rehydrated using a graded ethanol series, and subjected to immunohistochemical analysis. Prior to immunostaining for the SP and CGRP, sections were stained with hematoxylin and eosin (H\&E).

This study was approved by the Institutional Review Board of Chonbuk National University Hospital (IRB No. 2017-08-013).

\section{Immunohistochemistry}

Samples prepared as described above were treated with 3\% hydrogen peroxide in ice-cold methanol for 10 minutes to block endogenous peroxidase activity, rinsed in phosphate-buffered saline (PBS), and treated with $10 \%$ normal goat serum in PBS for 10 minutes to block nonspecific binding. Samples were then incubated overnight at $4^{\circ} \mathrm{C}$ with an anti-SP (1:500 titer; Abcam, Cambridge, UK) or anti-CGRP antibody (1:500 titer; Calbiochem, San Diego, CA, USA), washed twice with PBS, and treated with biotinylated secondary antibody (Vectastain) for 45 minutes at room temperature. Samples were then washed twice with PBS, treated with $0.03 \%$ diaminobenzidine tetrahydrochloride, and counterstained with modified Mayer's hematoxylin. Control sections were incubated with secondary antibody but not with primary antibody and processed in an identical manner.

\section{RESULTS}

On the H\&E stain, the well-developed large ceruminous glands (Fig. 1A) and small sebaceous glands near hair follicles (Fig. 1B) were found in the dermis. The secretory cell of a ceruminous gland was usually lined with high columnar cells resting on myoepithelial cells. High active columnar cells had secretory buds extending to the lumen of the tubule (Fig. 1A).

Results of the immunostaining for SP and CGRP in the epidermis and dermis of the EAC skin are summarized in Table 1. The degree of immunohistochemical positive density was determined using a 5-point scoring scale (reaction intensities: -, no

\section{H I}

- Calcitonin gene-related peptide (CGRP) and substance P (SP) were strongly stained in the ceruminous glands.

- Myoepithelial cells of the ceruminous gland were most intensely immunostained for both SP and CGRP.

- These peptides may participate in the regulation of secretion of ceruminous gland components.
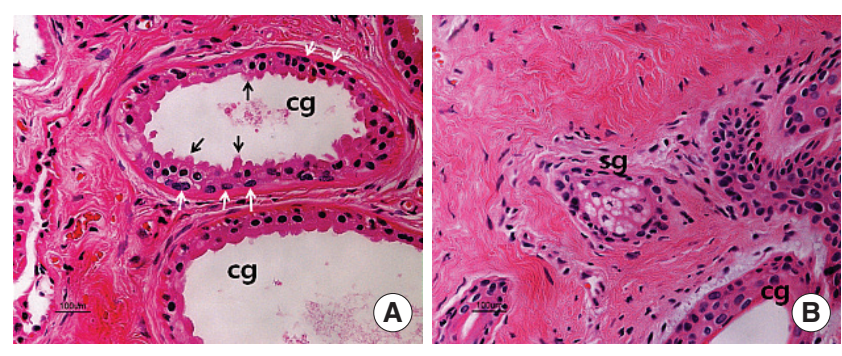

Fig. 1. Hematoxylin and eosin staining in the ceruminous gland $(A)$ and sebaceous gland (B). The ceruminous gland is a simple coiled tubular gland. The gland is lined by a single layer of secretory cells resting on myoepithelial cells (white arrows). The glandular epithelium is cuboidal or columnar and has protrusions (black arrows) extending to the lumen of the tubule. cg, ceruminous gland; sg, sebaceous gland $(H \& E, \times 200)$.

Table 1. Comparative study of immunostaining of SP and CGRP in the cartilaginous external auditory canal skin

\begin{tabular}{lcc}
\hline Variable & SP & CGRP \\
\hline Epidermis & & + \\
Conrnified cell & - & +++ \\
Suprabasal cell & - & + \\
Basal cell & - & \\
Dermis & - & ++ \\
Hair follicle & - & + \\
Sebaceous gland & - & +++ \\
Ceruminous gland & +++ & ++++ \\
Myoepithelial cell & ++++ & \\
\hline
\end{tabular}

Reaction intensities: -, no staining; +, mild; ++, moderate; +++, strong; ++++, very strong.

SP, substance P; CGRP, calcitonin gene-related peptide.

staining; +, mild; ++, moderate; +++, strong; ++++, very strong).

SP immunoreactivity was not detected in the epithelium of EAC skin (Fig. 2A, B) and sebaceous glands (Fig. 2D). In the ceruminous glands, SP-positive staining was found in both acini and myoepithelial cells (Fig. 2C).

In the epithelium, CGRP immunoreactivity was detected in the suprabasal region, which contained granular and prickle cell layers (Fig. 3A, B). CGRP positive staining was located in the dermis, ceruminous glands, sebaceous glands, and to a lesser extent around hair follicles. Furthermore, in the ceruminous glands, perinuclear region of secretory cells was positively stained (Fig. 3C, D) and more extensive in the cytoplasm of myoepithelial cells (Fig. 3D).

The density of SP and CGRP staining was highest in myoepithelial cells (Figs. 2C, 3C, 3D) of the ceruminous glands.

\section{DISCUSSION}

The appendages of the skin are sweat glands, sebaceous glands, and hair. Glands of EAC skin are responsible for secreting lipids, 

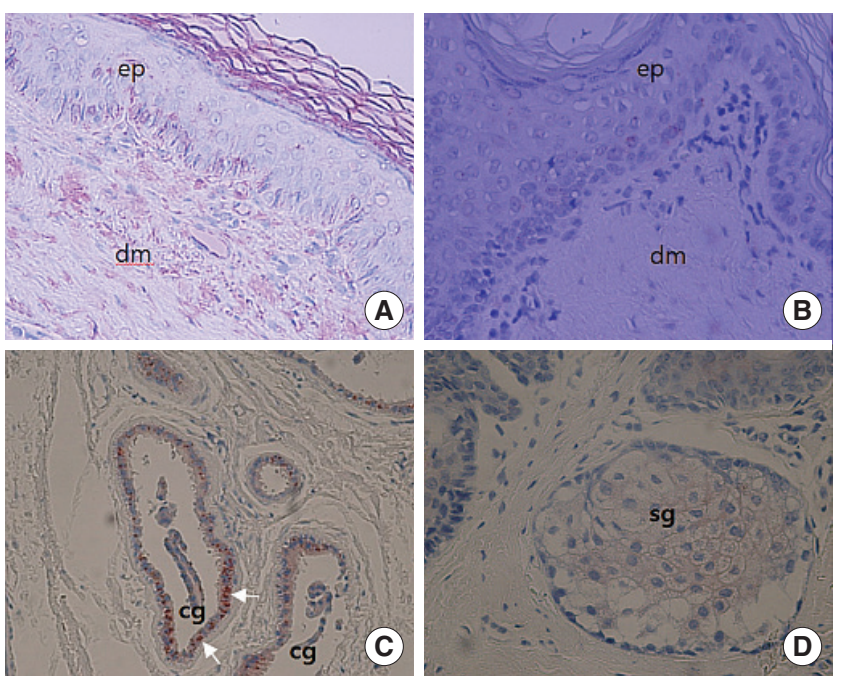

Fig. 2. Immunohistochemical labelling of substance P (SP) using an avidin-biotin peroxidase complex method in the external auditory canal skin. (A) SP positive staining is not noted in the epithelium of external auditory canal skin (X200), (B) higher magnification ( $X$ 400). (C) SP immunoreactivity is found in ceruminous gland (cg) especially in myoepithelial cells (white arrows) (×200). (D) SP immunoreactivity is not observed in sebaceous gland (sg) cells $(\times 200)$. ep, epithelium; dm, dermis.

peptides, and proteins, which are the components of cerumen and are important for maintaining the innate immunity of EAC $[1,5,6]$. Interestingly, the ceruminous glands are known to secrete several important antimicrobial proteins and peptides [26]. The ceruminous glands (modified sweat) in EAC skin are similar to the serous glands in the respiratory submucosa in terms of the biochemical protection. They afford and share several common properties. In particular, both contain numerous electron-dense secretory vesicles in the cytoplasm [9-11].

Ceruminous glands are located in the dermis deep to the sebaceous glands. They are simple coiled tubular glands. The glandular epithelium is cuboidal or columnar and has protrusions extending to the lumen of the tubule. In the active form, secretory cells are tall, with apical protrusions that were pinched off into the lumen of the gland. The ceruminous glands were found in a single layer of secretory cells resting upon one layer of myoepithelial cells, which surround the secretory cells as an outer layer of the tubule. Generally, the myoepithelial cells are found in the sweat glands, mammary glands, lacrimal glands, and salivary glands. Myoepithelial cells can function to squeeze-out the contents of the secretory cells into the duct.

Recently, some authors have suggested that ceruminous glands play a role in the protection of EAC skin since these glands contain antimicrobial factors $[5,6]$. The family of antimicrobial peptides secreted from these cells has been suggested to contribute to skin defenses, but the EAC skin has been in little attention [5].

In general, it has been known that skin gland secretion is un-
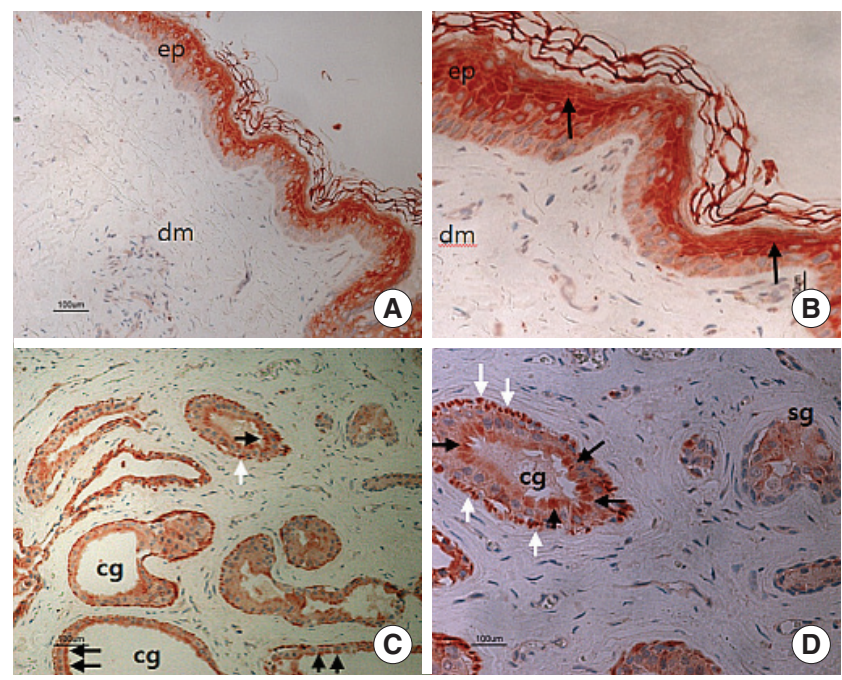

Fig. 3. Immunohistochemical labelling of calcitonin gene-related peptide (CGRP) using an avidin-biotin peroxidase complex method in the external auditory canal skin. (A) CGRP positive staining is prominently noted in the suprabasal portion of epithelium ( $\times 100)$. (B) Positive stained cells contain granular and prickle cell layers, in the epithelium (black arrows) (×200). (C, D) Reactions for CGRP were generally moderate in the secretory cells (black arrows), with their myoepithelial cells (white arrows) showing a stronger reaction. The CGRP staining in the sebaceous gland (sg) is somewhat weak, compared to other regions. ep, epithelium; dm, dermis; cg, ceruminous gland. $(C, \times 100 ; D, \times 200)$.

der neural control. The secretion of fluids and electrolytes is regulated by the parasympathetic system, whereas protein secretion is regulated by the sympathetic system. SP and CGRP have been known as a potent glandular fluid secretagogue [12] and to play a role in the gland secretion induced by harmful stimuli [8]. However, little is known about the secretagogues of EAC skin glands. It is suspected that the interrelation between antimicrobial materials and secretagugues has clinical significance in various EAC skin diseases. Moreover, it remains unclear whether SP and/or CGRP is expressed in the cells of EAC skin.

In the present study, the results of immunohistochemical analysis revealed that SP and CGRP were present in the cytoplasm of ceruminous gland cells, but not or sparse in sebaceous gland cells. Myoepithelial cells were intensely immunostained for both peptides. These findings indicated that SP and CGRP are expressed in the gland cells of EAC skin and suggest that these relate to the ceruminous gland secretion, in turn, those act to nullify pathogenic insults under normal conditions.

This is the first report of the presence of SP and CGRP in the gland cells of normal human EAC skin. The SP and CGRP immunoreactivity patterns observed in our study indicate that these secretary factors may participate in the regulation of secretion of cerumen components. These observations may provide important implications for EAC skin disorders. Further research into the secretion of antimicrobial proteins and peptides 
using these secretagogues is needed for increased understanding of the innate immune protection in the EAC skin.

\section{CONFLICT OF INTEREST}

No potential conflict of interest relevant to this article was reported.

\section{ACKNOWLEDGMENTS}

This paper was supported by the research fund of Chonbuk National University and a fund of the Biomedical Institute, Chonbuk National University Hospital (CUH 2015-0038).

\section{REFERENCES}

1. Main T, Lim D. The human external auditory canal, secretory system: an ultrastructural study. Laryngoscope. 1976 Aug;86(8):1164-76.

2. Ogawa H. Immunocytological localization of lysozyme in human skin. J Dermatol. 1975 Mar;2(1):45-50.
3. Harder J, Bartels J, Christophers E, Schroder JM. A peptide antibiotic from human skin. Nature. 1997 Jun;387(6636):861.

4. Ali RS, Falconer A, Ikram M, Bissett CE, Cerio R, Quinn AG. Expression of the peptide antibiotics human beta defensin- 1 and human beta defensin-2 in normal human skin. J Invest Dermatol. 2001 Jul; 117(1):106-11.

5. Yoon YJ, Park JW, Lee EJ. Presence of hBD-1 and hBD-2 in human cerumen and external auditory canal skin. Acta Otolaryngol. 2008 Aug;128(8):871-5.

6. Yoon YJ, Lee EJ. Spatial distribution of antimicrobial peptides and mast cells in the skin of the external auditory canal. J Laryngol Otol. 2011 Nov;125(11):e6.

7. Jankowski A, Kapusta E, Nowacka B. On the bacteriostatic or bactericidal function of ceruminous glands secretion. Otolaryngol Pol. 1992;46(6):557-60.

8. Church MK, Lowman MA, Robinson C, Holgate ST, Benyon RC. Interaction of neuropeptides with human mast cells. Int Arch Allergy Appl Immunol. 1989;88(1-2):70-8.

9. Ansel JC, Brown JR, Payan DG, Brown MA. Substance P selectively activates TNF-alpha gene expression in murine mast cells. J Immunol. 1993 May;150(10):4478-85.

10. Tandler B, Phillips CJ. Structure of serous cells in salivary glands. Microsc ResTech. 1993 Sep;26(1):32-48.

11. Jeffery PK. Morphologic features of airway surface epithelial cells and glands. Am Rev Respir Dis. 1983 Aug;128(2 Pt 2):S14-20.

12. Haxhiu MA, Haxhiu-Poskurica B, Moracic V, Carlo WA, Martin RJ. Reflex and chemical responses of tracheal submucosal glands in piglets. Respir Physiol. 1990 Dec;82(3):267-77. 